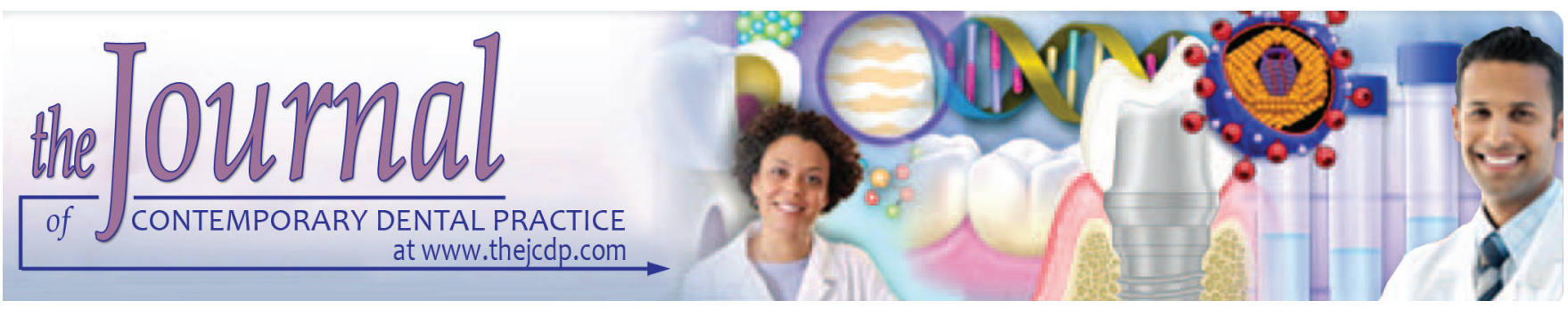

\title{
Immunohistochemical Detection of Proliferative Marker Ki-67 in Benign and Malignant Salivary Gland Tumors
}

\author{
${ }^{1}$ Smita Bussari, ${ }^{2}$ Sindhu M Ganvir, ${ }^{3}$ Manish Sarode, ${ }^{4}$ Prabhakar A Jeergal, ${ }^{5}$ Anjum Deshmukh, ${ }^{6}$ Himanshu Srivastava
}

\begin{abstract}
Introduction: Salivary gland tumors are the most histologically heterogeneous group of tumors with the greatest diversity of morphologic features among their cells and tissues. The present study was aimed at assessing the validity of $\mathrm{Ki}-67$, a cell proliferation marker, as a prognostic factor in benign and malignant salivary gland tumors and to study whether it is related to age, sex, anatomical site, and size of the lesion in salivary gland tumors.
\end{abstract}

Materials and methods: A retrospective study consisted of benign salivary gland tumors (BSGTs) $(n=15)$, malignant salivary gland tumors $(n=18)$, and normal salivary gland parenchyma ( $n=15)$.

Results: There was a significant difference of Ki-67 labeling index (LI, \%) in normal salivary gland parenchyma, BSGTs, and malignant salivary gland tumors. The Ki-67 LI (\%) in normal salivary gland parenchyma is negligible $(0.27 \pm 0.31 \%)$, whereas malignant salivary gland tumors showed very high Ki-67 LI (\%) of $18.79 \pm 18.06 \%$ compared with BSGTs being $0.76 \pm 2.02 \%$. There was a significant correlation statistically of mean \pm standard deviation (SD) of $\mathrm{Ki}-67 \mathrm{LI}(\%)$ with the age of the patients being the maximum $(32.68 \pm 15.87 \%)$ in the 50 to 59 years age group, whereas sex, site of the lesion, and size of the lesion in salivary gland tumors had no significant correlation.

\footnotetext{
${ }^{1,5}$ Department of Oral Pathology and Microbiology, M. A. Rangoonwala College of Dental Sciences \& Research Centre Pune, Maharashtra, India

${ }^{2}$ Department of Oral Pathology and Microbiology, Government Dental College \& Hospital, Nagpur, Maharashtra, India

${ }^{3}$ Department of Oral Pathology and Microbiology, Guru Gobind Singh College of Dental Science \& Research Centre, Burhanpur Madhya Pradesh, India

${ }^{4}$ Department of Oral Pathology and Microbiology, Sudha Rustagi College of Dental Sciences \& Research, Faridabad, Haryana India

${ }^{6}$ Department of Oral Pathology and Microbiology, Aditya Dental College \& Hospital, Beed, Maharashtra, India

Corresponding Author: Smita Bussari, Flat No. 2105, Tower 33, Amanora Park Town, Hadapsar, Pune, Maharashtra, India Phone: +919881107615, e-mail: drsmitabussari@gmail.com
}

Conclusion: The Ki-67 is a useful marker for assessing proliferative potential of tumors.

Clinical significance: The $\mathrm{Ki}-67 \mathrm{LI} \%$ can be used as a reliable adjuvant diagnostic tool to differentiate between the subtypes and grading of certain malignant tumors, such as mucoepidermoid carcinoma (MEC), adenoid cystic carcinoma (AdCC), and acinic cell carcinoma (AcCC), which are usually difficult to diagnose on histopathological criteria alone.

Keywords: Immunohistochemistry, Ki-67, Salivary gland neoplasms.

How to cite this article: BussariS, GanvirSM, SarodeM, Jeergal PA, Deshmukh A, Srivastava H. Immunohistochemical Detection of Proliferative Marker Ki-67 in Benign and Malignant Salivary Gland Tumors. J Contemp Dent Pract 2018;19(4):375-383.

Source of support: Nil

Conflict of interest: None

\section{INTRODUCTION}

Salivary gland tumors are reported to represent between 1 and $5 \%$ of all head and neck tumors and are either benign or malignant tumors. Tumors of the salivary gland constitute an important area in the field of oral and maxillofacial pathology. ${ }^{1}$ Of all the tissues in the human body, perhaps the salivary glands have the most histologically heterogeneous group of tumors and the greatest diversity of morphologic features among their cells and tissues.

Salivary gland tumors often pose considerable difficulty in differential diagnostic and prognostic assessment based on histomorphologic criteria alone due to overlapping clinicopathologic features, and tumors in the same type of classification schedule exhibit different clinical courses. ${ }^{2}$

Immunohistochemistry is an effective adjuvant to histopathological diagnosis and is based on hematoxylin and eosin (H\&E)-stained sections in the majority of equivocal tumor cases, and helps in the establishment of definitive diagnosis or confirmation of H\&E-stained sections. ${ }^{3}$ 
The Ki-67 human nuclear antigen is expressed during the $G_{1}, S, G_{2}$, and $M$ phases in the cell cycle, but is absent in the quiescent $G_{0}$ phase in tissue sections. The antibody raised against the $\mathrm{Ki}-67$ antigen has been used as simple, rapid, reliable means of evaluating growth fraction of normal and neoplastic cell populations. ${ }^{4,5}$

The purpose of the study was to assess the validity of $\mathrm{Ki}-67$, a cell proliferation marker, as a prognostic factor in benign and malignant salivary gland tumors and study whether it is related to age, sex, anatomical site, and size of the lesion in salivary gland tumors.

\section{MATERIALS AND METHODS}

\section{Tissue Sample}

Formalin-fixed, paraffin-embedded tissue blocks of 33 cases of salivary gland tumors and other 15 cases of normal salivary gland parenchyma were retrieved from the Histopathology Registry of Government Dental College and Hospital, Nagpur, India. All the samples were examined on H\&E-stained sections and classified as per criteria given by the World Health Organization (WHO) international classification of salivary gland tumors in 2005. The present study was approved by the Institutional Ethical Committee of the Government Dental College and Hospital, Nagpur, Maharashtra, India.

The study group of 33 cases of salivary gland tumors comprised 15 cases of BSGTs and 18 cases of malignant salivary gland tumors. The control group comprised 15 cases of normal salivary gland parenchyma from buccal mucosa, labial mucosa, and palate.

Clinical data including gender, age, tumor size, and location of the lesion were collected by reviewing the pathology records.

\section{Immunohistochemical Staining Procedure}

Sections of $4 \mu \mathrm{m}$ thickness were cut from formalin-fixed and paraffin-embedded tissue blocks and mounted on silane-coated glass slides. Paraffin sections were deparaffinized in xylene. Sections were rehydrated by transferring through descending grades of alcohol to water. Slides were heated in a pressure cooker for a total of 10 minutes in sodium citrate buffer $(0.01 \mathrm{M}, \mathrm{pH}$ 6.0) for antigen retrieval. Sections were incubated with peroxide block (3\% hydrogen peroxide in water) and washed with phosphate-buffered saline (PBS; pH 7.4, $0.05 \mathrm{M}$ ) three times for 5 minutes each. The sections were incubated with power block (contains casein and proprietary additives in PBS with $0.09 \%$ sodium azide) for 10 minutes at room temperature to block nonspecific immune reactions. Section was covered with ready-to-use mouse monoclonal antibody (anti-Ki-67 antigen, Biogenics) and incubated in a humidifying chamber at room temperature $\left(\approx 25^{\circ} \mathrm{C}\right)$ for
60 minutes and then kept overnight at $4^{\circ} \mathrm{C}$. Appropriate volume $(\approx 38 \mu \mathrm{L})$ of super enhancer reagent was added to cover the specimen according to tissue size to amplify the antigen-antibody reaction. Appropriate volume of poly-horse radish peroxide reagent was added to cover the specimen and incubated for 30 minutes at room temperature $\left(\approx 25^{\circ} \mathrm{C}\right)$ and rinsed thoroughly with PBS (pH 7.4, $0.05 \mathrm{M})$ thrice. Substrate solution [3,3'-diaminobenzidine (DAB)] was added to cover the specimen and incubated for 30 minutes at room temperature $\left(\approx 25^{\circ} \mathrm{C}\right)$ and rinsed with PBS (pH 7.4, 0.05 M) at least thrice. The sections were counterstained with Mayer's hematoxylin and mounted with coverslip using disterene polysterase xylene.

\section{Assessment of Immunohistochemically Stained Sections}

Immunohistochemically stained sections were assessed on the light microscope under $\times 400$ magnification and evaluated for Ki-67-positive cells. Cells were considered positive for the $\mathrm{Ki}-67$ antigen, if there was intranuclear DAB staining (brown color). All stained nuclei were scored positive regardless of the intensity of staining. Cells that lacked the clear nucleus were excluded. The counting protocol suggested by Hirabayashi ${ }^{6}$ and Okabe et $\mathrm{al}^{7}$ was followed. Five fields showing maximum Ki-67positive cells were selected at $\times 100$ magnification and a minimum of 1,000 tumor cells were counted in each section at $\times 400$ magnification. Computer-assisted cell counting was done using a cell image analyzer (Olympus BX51). The number of positively stained nuclei was expressed as a percentage of the total number of tumor cells counted per section, which is known as Ki-67 LI.

$$
\mathrm{Ki}-67 \mathrm{LI}=\frac{(\text { Number of Ki-67 positive nuclei) }}{(\text { Total number of tumor cells observed })} \times 100
$$

\section{Statistical Analysis}

Analysis of variance test was applied to compare the Ki-67 LI of normal salivary gland parenchyma, benign tumors of salivary gland, and malignant tumors of salivary gland. Analysis of the correlation between Ki-67 LI and clinicopathological factors (age, sex, site of lesion, and tumor size) was performed using unpaired t-test and Kruskal-Wallis test; $\mathrm{p}$-values were noted using software INTERCOOLED STATA version 8 . The $p$-values of $<0.05$ were regarded as statistically significant.

\section{RESULTS}

\section{Evaluation of H\&E-stained Sections}

A total of 33 cases of salivary gland tumors and 15 cases of normal salivary gland parenchyma were stained according to H\&E method to confirm the diagnosis and then classify salivary gland tumors according to the WHO international 
Table 1: Salivary gland tumors and normal salivary gland parenchyma based on H\&E staining method

\begin{tabular}{ll}
\hline Salivary gland tumors/control & Number of cases \\
\hline BSGTs & 15 \\
PA & 11 \\
ME & 2 \\
BCA & 2 \\
MSGTs & 18 \\
MEC & 6 \\
AdCC & 5 \\
AcCC & 3 \\
ACNos & 3 \\
PLGA & 1 \\
Control (NSGP) & 15 \\
\hline PA: PIeOmorphic adenoma
\end{tabular}

PA: Pleomorphic adenoma; MSGTs: Malignant salivary gland tumors; NSGP: Normal salivary gland parenchyma

classification of salivary gland tumors. The distribution of study group and controls is tabulated in Table 1.

\section{Correlation of Ki-67 LI (\%) with Histopathological Findings in Normal Salivary Gland Parenchyma and Salivary Gland Tumors}

\section{Evaluation of Mean of Ki-67 LI (\%) in Normal Salivary Gland Parenchyma}

In normal salivary gland parenchyma, the frequency of Ki-67-positive cells among the acinar cells and ductal cells including myoepithelial cells was calculated together. The mean \pm SD of Ki-67 LI (\%) in normal salivary gland parenchyma was $0.27 \pm 0.31 \%$, range $(0-0.88 \%)$.

\section{Evaluation of $\mathrm{Ki}-67 \mathrm{LI}(\%)$ in BSGTS}

Mean \pm SD of Ki-67 LI of 15 cases of BSGTs was $0.76 \pm$ $2.02 \%$, and there was negligible proliferative activity in pleomorphic adenoma and myoepithelioma (ME), whereas basal cell adenoma (BCA) showed little proliferative activity. As such, the overall proliferative activity was low in BSGTs (Figs 1 to 3).
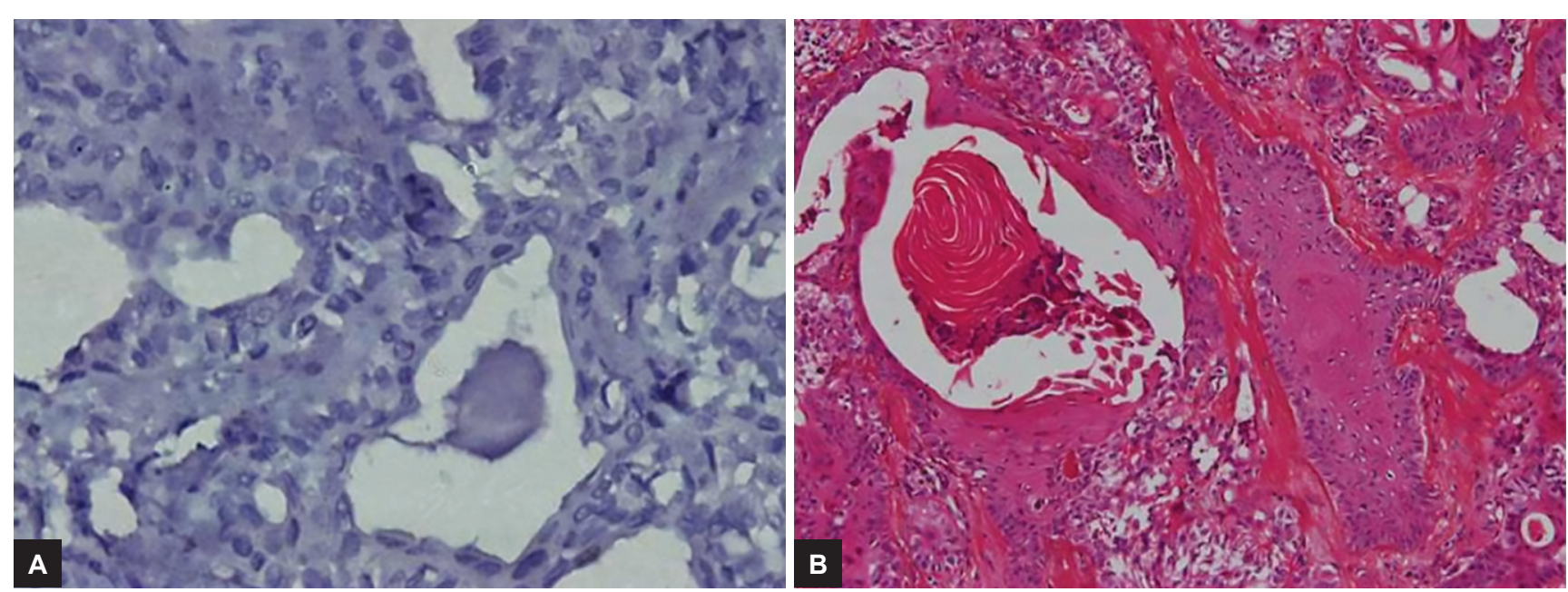

Figs 1A and B: (A) Pleomorphic adenoma (Ki-67-positive immunoreactivity in nuclei of tumor cells).

(B) Pleomorphic adenoma (H\&E stain, $\times 40$ magnification)

\section{Evaluation of Mean of Ki-67 LI (\%) in Malignant Salivary Gland Tumors}

Mean \pm SD of Ki-67 LI of 18 cases of malignant salivary gland tumors was $18.79 \pm 18.06 \%$, with a range of 0 to $59.09 \%$.

- Mean of Ki-67 LI (\%) in MEC

- Mean \pm SD of Ki-67 LI in 6 cases of MEC was $19.63 \pm 14.65 \%$ with range $(0-39.01 \%)$ and the subtypes of MEC showed mean \pm SD of Ki-67 LI in two cases of low-grade (LG) variant (Fig. 4), two cases of intermediate-grade (IG) variant (Fig. 5), and two cases of high-grade (HG) variant (Fig. 6) as $1.91 \pm 2.70,12.28 \pm 1.15$, and 34.88 $\pm 5.84 \%$ respectively. This showed the highest proliferative potential in the HG variant of MEC, which was statistically highly significant $(\mathrm{p}=0.0069)$.

- Mean of Ki-67 LI (\%) in AdCC

- Mean \pm SD of Ki-67 LI in 5 cases of AdCC was $5.38 \pm 5.78 \%$, range $(0-13.07 \%)$, and the subtypes of AdCC revealed mean \pm SD of Ki-67 LI in four cases of cribriform pattern (Fig. 7) of AdCC as $3.46 \pm 4.46 \%$ and one case of solid variant (Fig. 8) of AdCC was $13.07 \pm 0 \%$. This showed the much higher Ki-67 LI in solid variant (13.07 $\pm 0 \%$ ) than cribriform variant $(3.46 \pm 4.46 \%)$, and statistical analysis was not applied to this case due to less sample size.

- Mean of Ki-67 LI (\%) in AcCC

- Mean \pm SD of Ki-67 LI in 3 cases of AcCC was $24.41 \pm 24.72 \%$, range $(0-49.43 \%)$ and the subtypes of AcCC showed Ki-67 LI of one case of papillary cystic pattern and follicular pattern, and solid variant was zero $(0 \%), 23.79$, and $49.43 \%$ respectively.

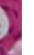




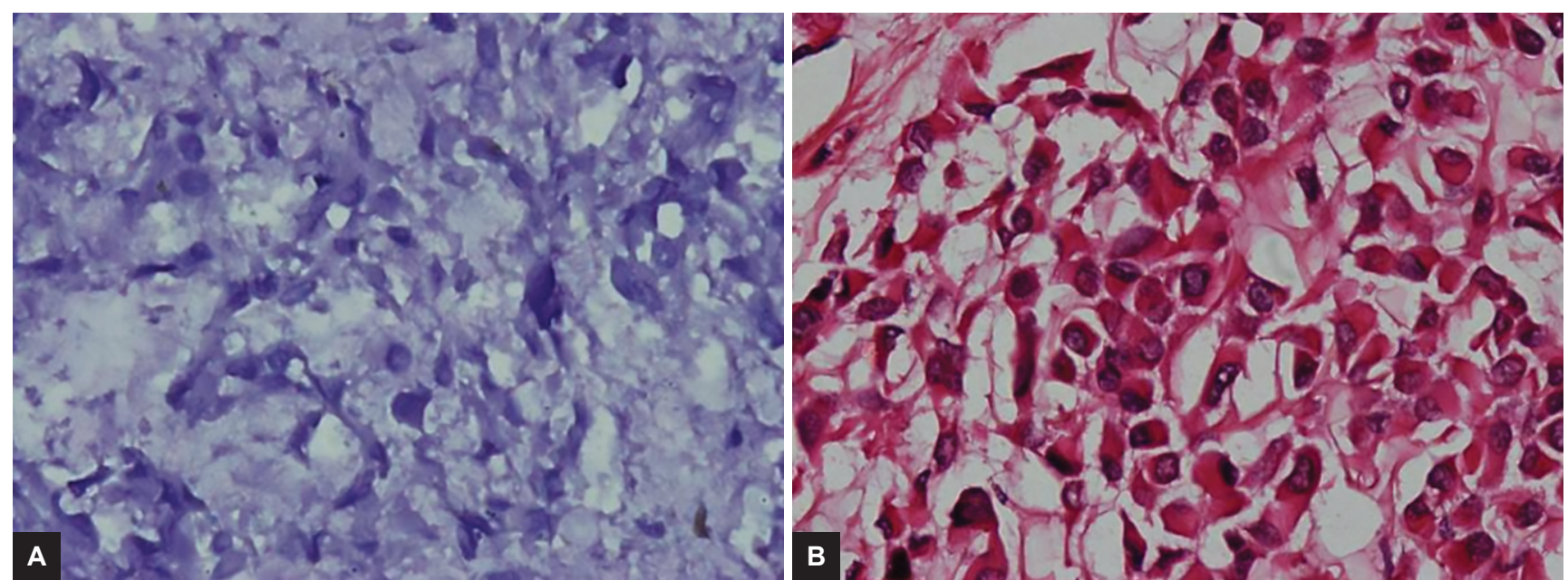

Figs 2A and B: (A) Myoepithelioma (Ki-67-positive immunoreactivity in nuclei of tumor cells). (B) Myoepithelioma (H\&E stain, $\times 40$ magnification)
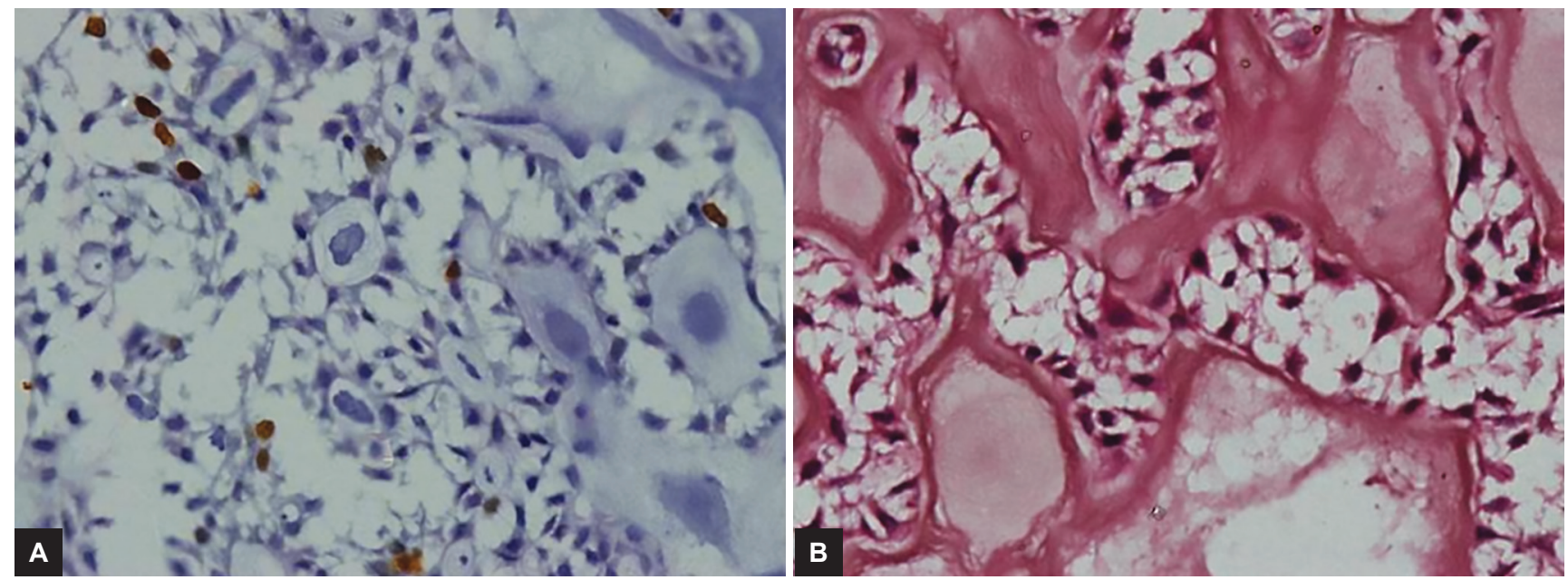

Figs 3A and B: (A) Basal cell adenoma (Ki-67-positive immunoreactivity in nuclei of tumor cells).

(B) Basal cell adenoma (H\&E stain, $\times 40$ magnification)

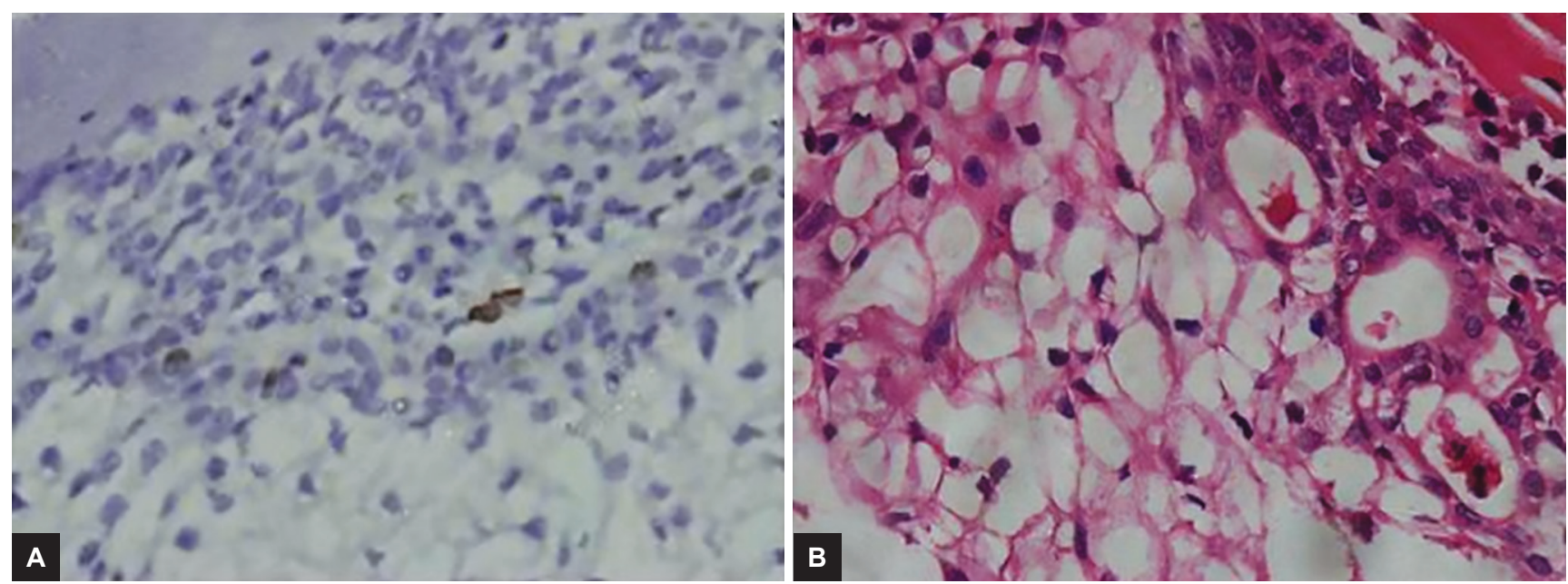

Figs 4A and B: (A) Mucoepidermoid carcinoma LG (Ki-67-positive immunoreactivity in nuclei of tumor cells).

(B) Mucoepidermoid carcinoma LG (H\&E stain, ×40 magnification) 

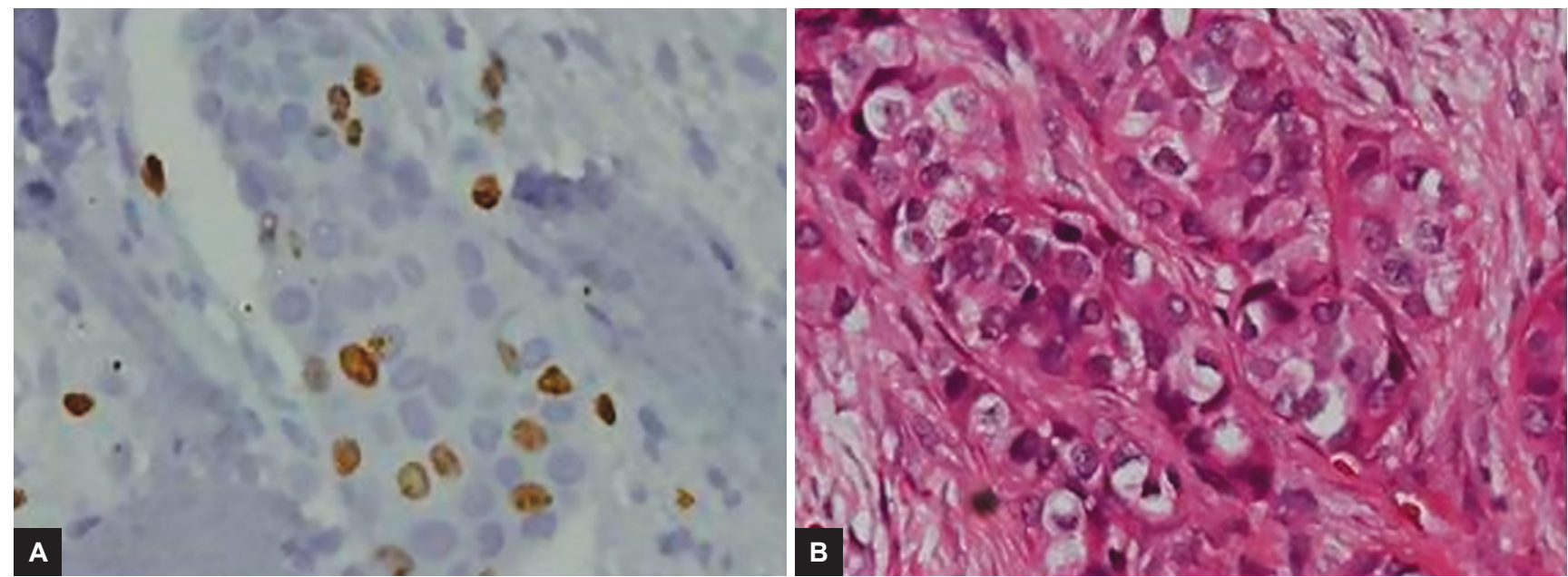

Figs 5A and B: (A) Mucoepidermoid carcinoma IG (Ki-67-positive immunoreactivity in nuclei of tumor cells). (B) Mucoepidermoid carcinoma IG (H\&E stain $\times 40$ magnification)
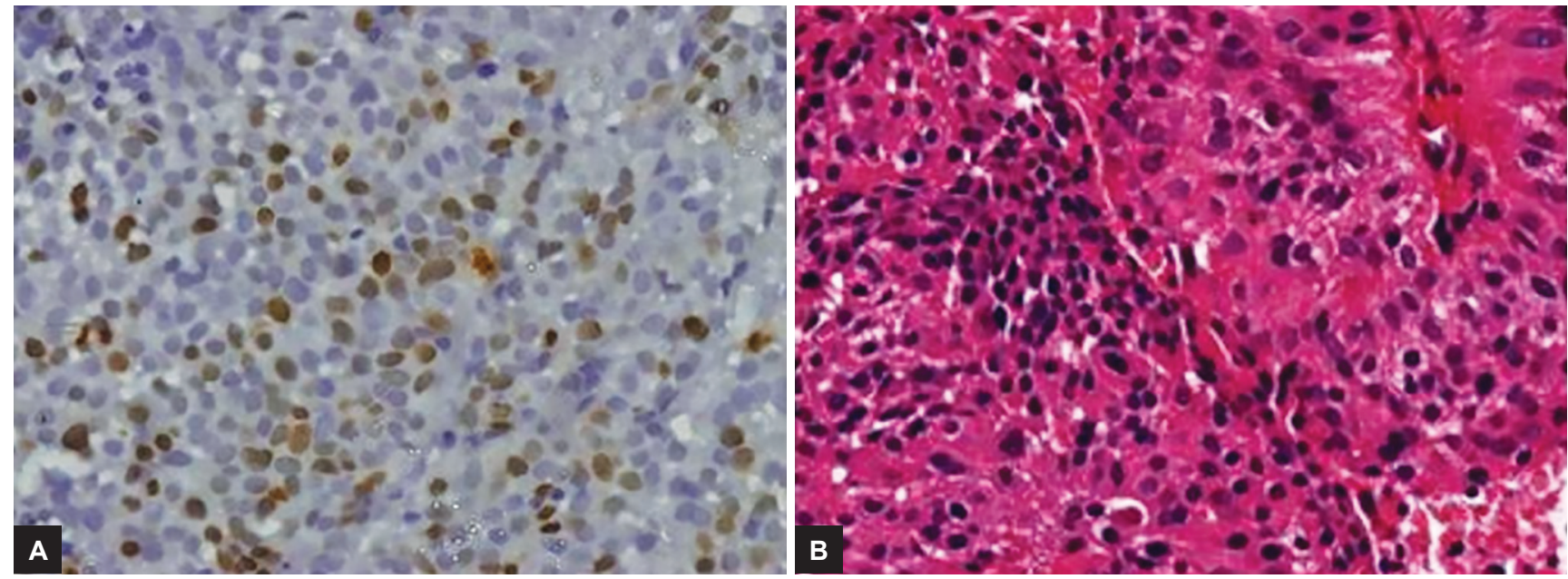

Figs 6A and B: (A) Mucoepidermoid carcinoma HG (Ki-67-positive immunoreactivity in nuclei of tumor cells). (B) Mucoepidermoid carcinoma HG (H\&E stain $\times 40$ magnification)
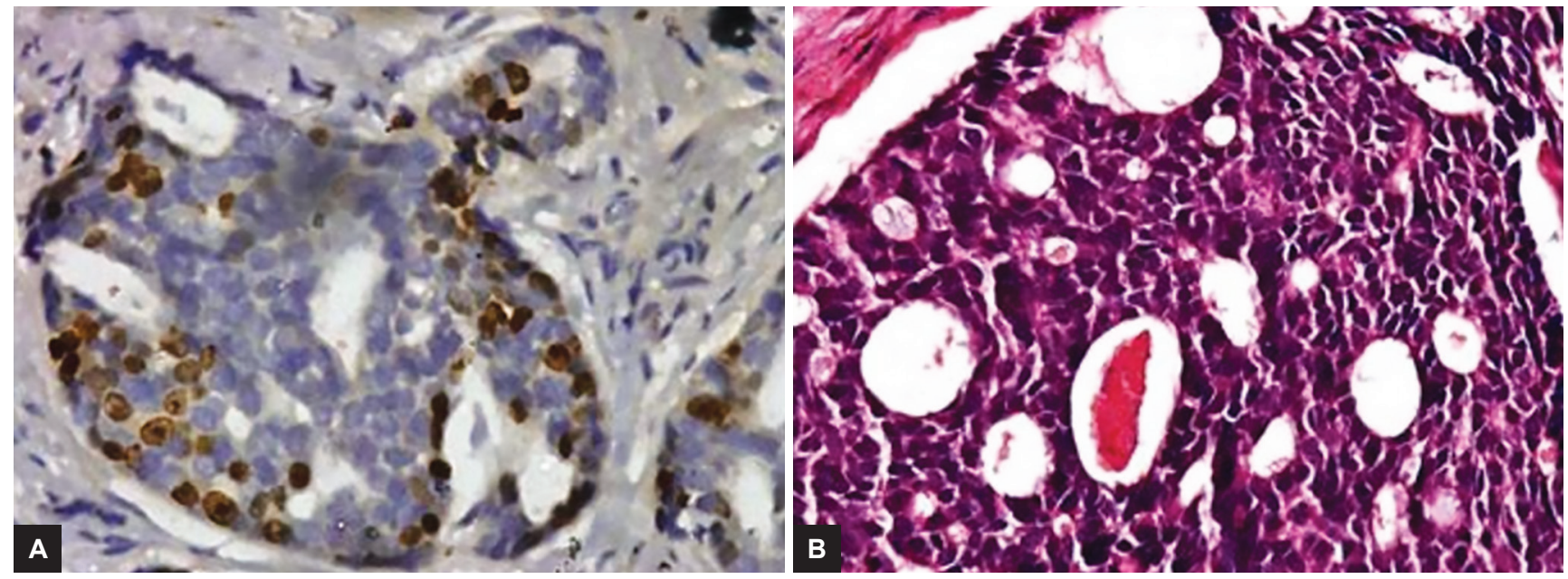

Figs 7A and B: (A) Adenoid cystic carcinoma cribriform pattern (Ki-67-positive immunoreactivity in nuclei of tumor cells). (B) Adenoid cystic carcinoma cribriform pattern (H\&E stain $\times 40$ magnification) 


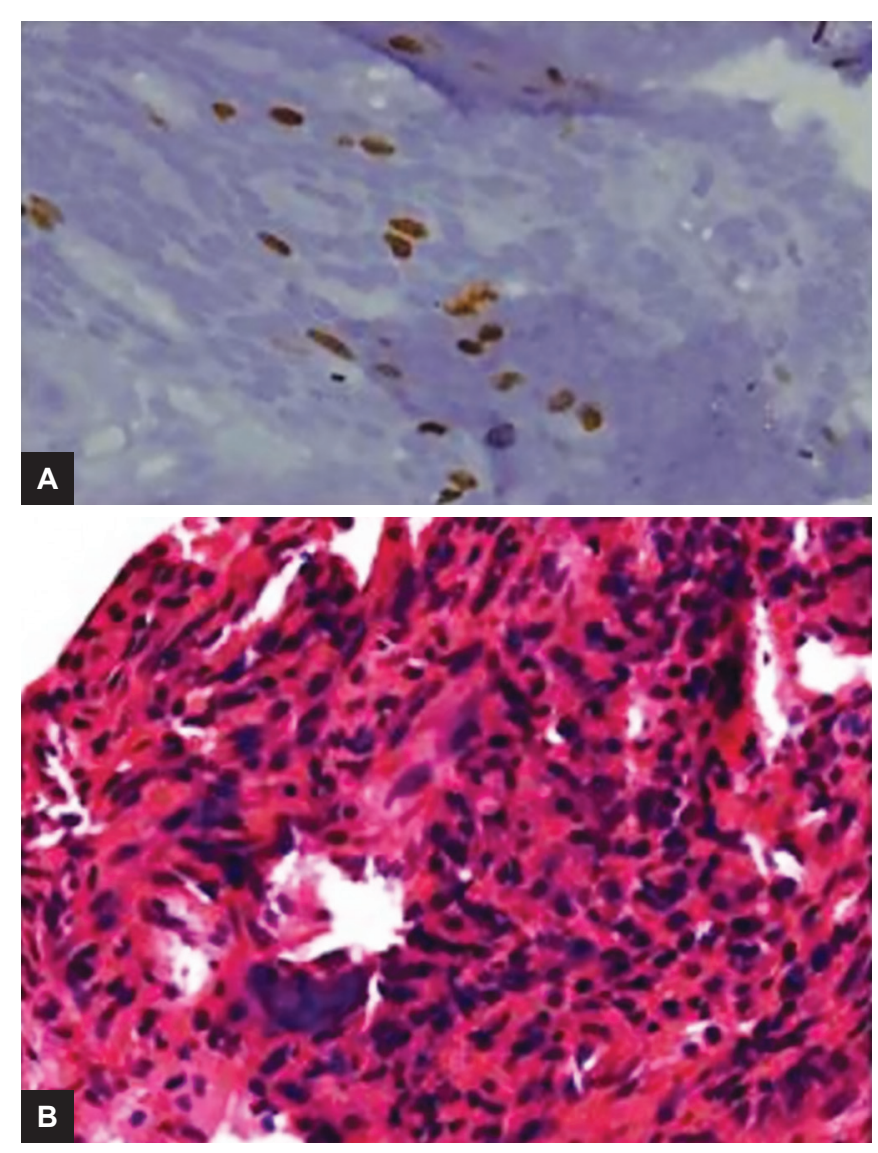

Figs 8A and B: (A) Adenoid cystic carcinoma solid pattern (Ki-67positive immunoreactivity in nuclei of tumor cells). (B)Adenoid cystic carcinoma solid pattern (H\&E stain, $\times 40$ magnification)

- Mean of Ki-67 LI (\%) in adenocarcinoma not otherwise specified (ACNos)

- Mean \pm SD of Ki-67 LI in three cases of ACNos was $26.99 \pm 8.81 \%$, range (17.87-35.45\%).

- Mean of Ki-67 LI (\%) in polymorphous LG adenocarcinoma (PLGA)

- Mean \pm SD of Ki-67 LI in one case of PLGA of HG variant was $59.09 \pm 0$.

\section{Comparison of Mean of Ki-67 LI (\%) of Normal Salivary Gland Parenchyma with BSGTs and Malignant Salivary Gland Tumors}

Difference of the mean \pm SD of Ki-67 LI between the normal salivary gland parenchyma, BSGTs, and malignant salivary tumors was subjected to statistical analysis. This revealed a statistically nonsignificant correlation between normal salivary gland parenchyma and BSGTs (as p >0.05), but when mean \pm SD of Ki-67 LI of normal salivary gland parenchyma was compared with malignant salivary gland tumors, it was found to be highly significant (as $\mathrm{p}<0.01$ ). Similarly, high statistical significance was found between benign and malignant salivary gland tumors $($ as $\mathrm{p}<0.01)$ as shown in Graph 1.

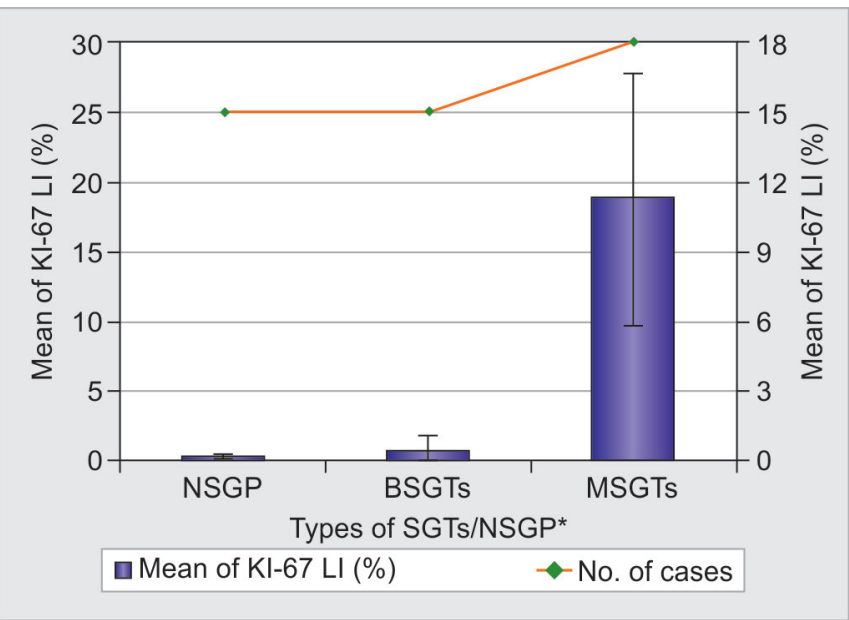

Graph 1: Comparison of mean of Ki-67 LI (\%) in BSGTs, malignant salivary gland tumor $v s$ normal salivary gland parenchyma* (total number of cases $=48$ )

\section{Correlation of Ki-67 LI (\%) with Clinical Parameters of Salivary Gland Tumors}

Among 33 cases of salivary gland tumors (benign and malignant), there was a significant correlation statistically of mean \pm SD of Ki-67 LI (\%) with the age of the patients being the maximum $(32.68 \pm 15.87 \%)$ in the 50 to 59 years age group, whereas sex, site of the lesion, and size of the lesion in salivary gland tumors had no significant correlation.

\section{DISCUSSION}

Keeping in view the diverse histomorphology, varied growth pattern, and varied behavior of salivary gland tumors, numerous studies have been performed seeking for prognostic factors. ${ }^{8-10}$ Immunohistochemistry is an effective adjunct to diagnosis based on H\&E-stained sections in the majority of equivocal tumor cases, through the establishment of definitive diagnosis or through confirmation of diagnosis on H\&E-stained sections. ${ }^{11}$ Cell proliferation, in particular, has been extensively studied. Detection of nuclear antigens related to cell cycle has made it possible to estimate cellular proliferation activity by immunohistochemical methods. ${ }^{10,12}$ The Ki- 67 antigen has been used as a simple, rapid, and reliable means of evaluating growth fraction. 13,14

In the present study, among 15 cases of normal salivary gland parenchyma, mean \pm SD of Ki-67 LI was 0.27 $\pm 0.31 \%$ and range was 0 to $0.88 \%$. Our findings are in accordance with the previous studies by Hirabayashi ${ }^{6}$ and Jia-Xuan et $\mathrm{al}^{15}$ and are quite on the higher side in the study of Murakami et $a{ }^{16}$ compared with our study. Probably, the study on frozen section and the sample size could be responsible.

In our study, all the pleomorphic adenomas were negative for Ki-67 immunoreactivity. This reveals the 
low proliferative activity in tumor. Thus, our findings are in agreement with those of Alves et al, ${ }^{17,18}$ whereas Hirabayashi, ${ }^{6}$ Jia-Xuan et al, ${ }^{15}$ and Vargas et $\mathrm{al}^{19}$ found a very low proliferative potential in pleomorphic adenoma ranging from 1.3 to $6.47 \%$. This variation in our findings with previous studies could be due to the variations in the histological features of pleomorphic adenoma, such as necrosis, hyalinization, increased abnormal mitotic activity, and stroma-rich lesions that may grow at a faster rate. These factors influence the proliferative activity in tumor as commented by Alves et al. ${ }^{17}$ In this study, ME was negative for the Ki-67 immunoreactivity, indicating extremely low proliferative rate. Nagao et $\mathrm{al}^{20}$ in their study, in Japan reported a range of Ki-67 LI from 0.9 to $9.1 \%$ in six cases of ME. Ferri et $\mathrm{al}^{21}$ found $\mathrm{Ki}-67 \mathrm{LI}$ as $1 \%$ in their study of one case of $\mathrm{ME}$. This shows that ME has very low proliferative rate as also found in our study.

Our study revealed mean $\pm \mathrm{SD}$ of $\mathrm{Ki}-67 \mathrm{LI}$ in BCA as $5.70 \pm 0.93 \%(5.04-6.36 \%)$. To the best of our knowledge, no reported study has investigated $\mathrm{Ki}-67$ expression in BCA.

In our study, among six cases of MEC, Ki-67 LI in HG variant was higher than IG and LG variants, i.e., mean of Ki-67 LI of HG > IG > LG. This difference in mean of Ki-67 LI $(\%)$ was statistically significant $(p=0.0069)$. Thus, our findings are in agreement with all the previous studies by Hicks and Flaitz, ${ }^{10}$ Okabe et al, ${ }^{7}$ Nguyen et al, ${ }^{22}$ Triantafillidou et $\mathrm{al}^{23}$ and Vargas et al. ${ }^{19}$ This suggests that the Ki-67 LI correlates very well with histopathological grading of MEC, and therefore, high Ki-67 LI indicates that the HG variant of MEC has the highest proliferative activity.

In the present study, among five cases of AdCC, Ki-67 LI (\%) of solid variant was higher than that of the cribriform pattern. Thus, our results are in accordance with lower values compared with Hirabayashi ${ }^{6}$ and Amoueian et al. ${ }^{24}$ These results suggest that the solid variant of AdCC has high proliferative activity than cribriform variant of AdCC.

The present study revealed that Ki-67 LI of solid variant of AcCC was greater than that of the follicular pattern, whereas the papillary cystic pattern showed negative expression for Ki-67 immunoreactivity. These results suggest that the differentiation of subtypes of AcCC is important as it reflects its proliferative activity and has its prognostic value. Till to date, no studies have reported the expression of $\mathrm{Ki}-67$ in the subtypes of AcCC, and, hence, further research to confirm these findings would be of value.

Three cases of ACNos had a mean of Ki-67 LI as 26.99 $\pm 8.81, \mathrm{SD} \%$. To the best of our knowledge, no reported study has investigated Ki-67 expression in ACNos.
In the present study of only one case of PLGA, the Ki-67 LI was 59.09\%, which had a higher value and the tumor showed HG malignancy on H\&E-stained section. Simpson et al, ${ }^{25}$ who reported two cases of PLGA with transformation to HG carcinoma, found that Ki-67 LI of 2 and 3\% was typical in LG areas, in contrast to 30 and $38 \%$ in $\mathrm{HG}$ elements. Darling et $\mathrm{al}^{26}$ noted an average of Ki-67-staining intensity as 6.2\% (0.2-14.3\%). Beltran et $\mathrm{al}^{27}$ reported a range of $\mathrm{Ki}-67 \mathrm{LI}$ from 0 to $6.74 \%$ with a mean of $1.24 \%$. Vargas et $\mathrm{al}^{19}$ noted the range of $\mathrm{Ki}-67 \mathrm{LI}$ in PLGA from 0 to $14.30 \%$ with a mean of $2.55 \pm 4.24 \%$. Disparity in the results could be due to HG malignancy of the present case of PLGA and less sample size. In our study, discrepancies in results reported relative to others may be related to factors, such as variability in primary antibodies selected, differences in immunohistochemistry protocols, varying methods of evaluating immunoreactivity, and limited case numbers.

\section{Comparison of Mean of Ki-67 LI (\%) of Normal Salivary Gland Parenchyma with BSGTs and Malignant Salivary Gland Tumors}

Expression of Ki-67-positive cells in malignant salivary gland tumors was very much comparable with other studies. However, mean \pm SD of BSGTs and normal salivary gland parenchyma was quite on higher side, especially normal salivary gland parenchyma in Murakami et $\mathrm{al}^{\prime} \mathrm{s}^{16}$ study compared with our study. Probably, the study on frozen section and sample size could be responsible.

No other literature studies have evaluated Ki-67 LI (\%) simultaneously in benign, malignant, and normal salivary gland parenchyma as per our knowledge.

\section{Correlation of Ki-67 LI (\%) with Clinical Parameters of Salivary Gland Tumors}

Among 33 cases of salivary gland tumors (benign and malignant), there was a significant correlation statistically of mean \pm SD of Ki- $67 \mathrm{LI}(\%)$ with the age of the patients being the maximum $(32.68 \pm 15.87 \%)$ in 50 to 59 years age group, whereas sex, site of the lesion, and size of the lesion in salivary gland tumors had no significant correlation.

Our findings are not in accordance to the study by Vargas et al ${ }_{1}^{19}$ who found no significant correlation with age of patients in their study of 62 cases of salivary gland tumors comprised of benign and malignant tumors. This variation could be due to less sample size in the present study.

Lim et a ${ }^{28}$ found a significant correlation between the Ki-67 expression and size of the tumor, i.e., Ki-67 LI (\%) increased with increase in the size of tumor in this study of 45 cases of malignant salivary gland tumors. Our study 
was not in line with Lim et al. ${ }^{28}$ This could be, as in our present study, due to correlation being irrespective of benign and malignant, whereas Lim et $\mathrm{al}^{28}$ has included only malignant salivary gland tumors.

\section{CONCLUSION}

Thus, it can be concluded that there is a definite difference in the Ki-67 LI (\%) of normal salivary gland parenchyma, BSGTs, and malignant salivary gland tumors, being highest in the malignant salivary gland tumors. Similarly, Ki-67 LI (\%) is also variable depending upon its grade as well as subtype of malignant salivary gland tumors.

\section{CLINICAL SIGNIFICANCE}

Although this technique is beneficial in differentiating between salivary gland parenchyma and benign and malignant salivary gland tumors, the overlapping of the Ki-67 LI (\%) between certain tumors prohibited the use of this technique as an absolute criteria in establishing a final diagnosis, but can be used as a reliable adjuvant diagnostic tool to differentiate between the subtypes and grading of certain malignant tumors, such as MEC, AdCC, and AcCC, which are usually difficult to diagnose on histopathological criteria alone.

Since it is a useful marker for assessing the proliferative potential of tumors, the prognosis of patients can definitely be predicted.

\section{REFERENCES}

1. Neville, BW.; Damm, DD.; Allen, CM.; Bouquot, JE. Oral and maxillofacial pathology. 2nd ed. Philadelphia (PA): W.B. Saunders Company; 2004. pp. 406-430.

2. Skálová A, Leivo I. Cell proliferation in salivary gland tumors. Gen Diagn Pathol 1996 Jun;142(1):7-16.

3. Jordan RC, Daniels TE, Greenspan JS, Regezi JA. Advanced diagnostic methods in oral and maxillofacial pathology. Part II: immunohistochemical and immunofluorescent methods. Oral Surg Oral Med Oral Pathol Oral Radiol Endod 2002 Jan;93(1):56-74.

4. du Manoir S, Guillaud P, Camus E, Seigneurin D, Brugal G. Ki-67 labeling in postmitotic cells defines different ki-67 pathways within the 2c compartment. Cytometry 1991;12(5): 455-463.

5. Bhaskar, SN. Salivary glands. In: Orban's oral histology and embryology. 10th ed. New Delhi: CBS Publishers; 1990. pp. 328-361.

6. Hirabayashi S. Immunohistochemical detection of DNA topoisomerase Type II alpha and ki-67 in adenoid cystic carcinoma and pleomorphic adenoma of the salivary gland. J Oral Pathol Med 1999 Mar;28(3):131-136.

7. Okabe M, Inagaki H, Murase T, Inoue M, Nagai N, Eimoto T. Prognostic significance of p27 and ki-67 expression in mucoepidermoid carcinoma of the intraoral minor salivary gland. Mod Pathol 2001 Oct;14(10):1008-1014.
8. Skalova A, Lehtonen H, von Boguslawsky K, Leivo I. Prognostic significance of cell proliferation in mucoepidermoid carcinomas of the salivary gland: clinicopathological study using MIB 1 antibody in paraffin sections. Hum Pathol 1994 Sep;25(9):929-935.

9. Nordgård S, Franzén G, Boysen M, Halvorsen TB. Ki-67 as a prognostic marker in adenoid cystic carcinoma assessed with the monoclonal antibody MIB1 in paraffin sections. Laryngoscope 1997 Apr;107(4):531-536.

10. Hicks J, Flaitz C. Mucoepidermoid carcinoma of salivary glands in children and adolescents: assessment of proliferation markers. Oral Oncol 2000 Sep;36(5):454-460.

11. Mason DY, Gatter KC. The role of immunocytochemistry in diagnostic pathology. J Clin Pathol 1987 Sep;40(9):1042-1054.

12. Zhu Q, Tipoe GL, White FH. Proliferative activity as detected by immunostaining with ki-67 and proliferating cell nuclear antigen in benign and malignant epithelial lesions of the human parotid gland. Anal Quant Cytol Histol 1999 Aug;21(4): 336-342.

13. Kill IR. Localisation of the ki-67 antigen within the nucleolus. Evidence for a fibrillarin-deficient region of the dense fibrillar component. J Cell Sci 1996 Jun;109(Pt 6):1253-1263.

14. Scragg MA, Johnson NW. Epithelial cell kinetics-a review of methods of study and their application to oral mucosa in health and disease. Part A. Methods for studying cell proliferation and some sources of variation. J Oral Pathol 1980 Nov;9(6):309-341.

15. Jia-Xuan QI, Sheng-Rong ZH, Sudhott H, Hildman $H$. Expression of Ki-67, PCNA in parotid tumors. J US China Med Sci 2008;5:37-42.

16. Murakami M, Othani I, Hojo H, Wakasa H. Immunohistochemical evaluation with Ki-67: an application to salivary gland tumours. J Laryngol Otol 1992 Jan;106(1):35-38.

17. Alves FA, Perez DE, Almeida OP, Lopes MA, Kowalski LP. Pleomorphic adenoma of the submandibular gland: clinicopathological and immunohistochemical features of 60 cases in Brazil. Arch Otolaryngol Head Neck Surg 2002 Dec;128(12):1400-1403.

18. Alves FA, Pires FR, De Almeida OP, Lopes MA, Kowalski LP. PCNA, ki-67 and p53 expressions in submandibular salivary gland tumours. Int J Oral Maxillofac Surg 2004 Sep;33(6): 593-597.

19. Vargas PA, Cheng Y, Barrett AW, Craig GT, Speight PM. Expression of $\mathrm{mcm}-2, \mathrm{ki}-67$ and geminin in benign and malignant salivary gland tumours. J Oral Pathol Med 2008 May;37(5):309-318.

20. Nagao T, Sugano I, Ishida Y, Tajima Y, Matsuzaki O, Konno A, Kondo Y, Nagao K. Salivary gland malignant myoepithelioma: a clinicopathologic and immunohistochemical study of ten cases. Cancer 1998 Oct;83(7):1292-1299.

21. Ferri E, Pavon I, Armato E, Cavaleri S, Capuzzo P, Ianniello F. Myoepithelioma of a minor salivary gland of the cheek: case report. Acta Otorhinolaryngol Ital 2006 Feb;26(1):43-46.

22. Nguyen LH, Black MJ, Hier M, Chauvin P, Rochon L. HER2/ neu and Ki-67 as prognostic indicators in mucoepidermoid carcinomid carcinoma of salivary glands. J Otolaryngol 2003 Oct;32(5):328-331.

23. Triantafillidou K, Dimitrakopoulos J, Iordanidis F, Koufogiannis D. Mucoepidermoid carcinoma of minor salivary glands: a clinical study of 16 cases and review of the literature. Oral Dis 2006 Jul;12(4):364-370. 
24. Amoueian S, Saghafi S, Farhadi F, Tohidi E, Sadegi L. Immunohistochemical assessment of Ki-67 expression in adenoid cystic carcinoma of the salivary glands. Iranian J Basic Med Sci 2007 Apr;10(2):84-89.

25. Simpson RH, Pereira EM, Ribeiro AC, Abdulkadir A, ReisFilho JS. Polymorphous low-grade adenocarcinoma of the salivary glands with transformation to high-grade carcinoma. Histopathology 2002 Sep;41(3):250-259.

26. Darling MR, Schneider JW, Muller CJ. Differential immunohistochemical staining in polymorphous low grade adenocarcinoma and adenoid cystic carcinoma: Bcl-2, MIB-1 and TP53. Oral Biosci Med 2004 Jul;1(3):207-211.

27. Beltran D, Faquin WC, Gallagber G, August M. Selective immunohistochemical comparison of polymorphous lowgrade adenocarcinoma and adenoid cystic carcinoma. J Oral Maxillofac Surg 2006 Mar;64(3):415-423.

28. Lim JJ, Kang S, Lee MR, Pai HK, Yoon HJ, Lee JI, Hong SP, Lim CY. Expression of vascular endothelial growth factor in salivary gland carcinomas and its relation to p53, ki-67 and prognosis. J Oral Pathol Med 2003 Oct;32(9):552-561. 